\title{
Caracterización del sistema de innovación para la ganadería familiar en la región de la Sierras del Este de Uruguay
}

\section{Characterization of the innovation system for family livestock farmers in the Sierras del Este region of Uruguay}

Jorge Álvarez ${ }^{1 *}$, Pablo Areosa ${ }^{1}$, Carlos Molina ${ }^{1}$, Norberto Rodríguez ${ }^{1}$, Pedro de Hegedus ${ }^{1}$

\section{RESUMEN}

En el proyecto "Co-innovación para el desarrollo de la ganadería familiar en la región de Sierra del Este", se caracterizó el sistema de innovación regional. Se realizaron entrevistas a informantes calificados y mapeos de actores con las tres organizaciones de productores (OP) integradas al proyecto. Los principales actores del sistema de innovación son el INIA Treinta y Tres, el Instituto Plan Agropecuario y las OP involucradas, la SFR\&I de Maldonado, la SR Garzón y la AFR Las Cañas. Los agentes de la investigación (INIA y Fagro-UdelaR) presentan poco vínculo con las OP. Los dispositivos para captar demandas desde los productores no funcionan adecuadamente. Desde la producción no aparece claro la presencia de interlocutores organizados, que interactúen con el sistema. Es necesario mejorar los niveles de capital social de las OP y su capacidad de participación en estructuras de decisión. La Mesa de Desarrollo Rural puede jugar un papel importante.

Palabras claves: Extensión, productores ganaderos familiares, sistema de innovación.

\begin{abstract}
Within the project "Co-innovation for the development of family livestock in the Sierra del Este region", a characterization of the innovation system was carried out. Data came from interviewing qualified informants and stakeholder mapping through rapid participatory diagnostic workshops conducted with partner farmer organizations (FO) belonging to the project. The main actors of the innovation system are the INIA Treinta y Tres, the Instituto Plan Agropecuario and the FO, which in the project area are SFR\&I de Maldonado, SR Garzón and AFR Las Cañas. Research agents (INIA and Fagro-UdelaR) appear with little interaction with FO. Likely, mechanisms to identify demands from livestock farmers do not work in the best way. From the demand side it is not clear the presence of organized interlocutors, who interact within the system. Social capital needs to be improved and also their capacity to participate in decision-making structures as the "Mesa de Desarrollo Rural".
\end{abstract}

Keywords: Extension, family livestock farmers, innovation system.

\footnotetext{
${ }^{1}$ Departamento de Ciencias Sociales de Facultad de Agronomía, Universidad de la República de Uruguay, Avenida Eugenio Garzón N 780, Montevideo, Uruguay.

Esta investigación fue realizada en el marco de un proyecto financiado por el Fondo de Promoción de Tecnologías Agropecuarias (FPTA) del Instituto Nacional de Investigaciones Agropecuarias del Uruguay.

"Autor de correspondencia.E-mail: jalvarez@fagro.edu.uy
} 


\section{I.INTRODUCCIÓN}

Los productores ganaderos familiares de las Sierras del Este, población objetivo de este trabajo, han sido caracterizados como un grupo de productores, dentro del contexto nacional, que presentan indicadores productivos (porcentaje de preñez, edad de entore o encarnerada, porcentajes y pesos de destete, entre otros) que pueden ser mejorados por la aplicación de tecnologías existentes (INIA, 2015). En la Tabla 1 se presentan datos censales (DIEA, 2013) de tres agrupamientos de productores ganaderos familiares. La primera columna refiere a todo el país, la segunda, a la región de Sierra del Este, que comprende los departamentos de Maldonado, Treinta y Tres, Rocha y Lavalleja, mientras que la final a los productores localizados en el departamento de Maldonado.

Los productores ganaderos familiares ubicados en el departamento de Maldonado representan en 5,3\% de este grupo dentro del país, explotan predios de mayor tamaño (la mediana es de 82 ha), similar en tamaño al agrupamiento de Sierras del Este y manejan un rodeo de cría vacuna de 44 vacas. Hacen una ganadería mixta con una mayor proporción de ovinos que sus pares nacionales y regionales y usan el suelo con un porcentaje similar de campo natural, el cual representa 3 de cada 4 hectáreas aplicadas al pastoreo.

Estos titulares en promedio tienen 55 años de edad, 2 años mayores que sus pares nacionales y regionales. Tienen un nivel educativo más alto, residen en menor porcentaje en los establecimientos, y solo 3 de cada 10 recibe algún tipo de asistencia técnica, 10 puntos porcentuales menos que el grupo nacional.

$\mathrm{Al}$ intentar explicar el problema del rezago productivo, (Gómez y Saravia, 2016) identifican cinco determinantes que explican la tasa de adopción de tecnologías disponibles. Según los autores estos son: la escala de producción (tamaño de las explotaciones o de los rodeos), la disponibilidad de instalaciones para el manejo animal, la asistencia a reuniones técnicas, la percepción de que el negocio en ganadería pasa por producir más y el tener asistencia técnica. Las últimas tres determinantes constituyen aspectos directamente asociados a la forma en cómo se gestiona el conocimiento y como circulan y se vuelven disponibles las innovaciones tecnológicas.

A partir del paradigma de cambio técnico lineal (la difusión de innovaciones de E. Rogers), los enfoques teóricos recorrieron dos vertientes. La visión de $\mathrm{P}$. Freire desde A. Latina que apostaba a una educación critica enmarcada en cambios estructurales (continuada en lo que se denomina Investigación Acción Participativa) y la visión sistémica del cambio técnico. En esta corriente podemos identificar los aportes de N. Roling en la década del 80 en la Universidad de Wageningen, que constituyen una etapa previa a lo que actualmente se denomina sistemas de innovación. Estos aportes se estructuraron en torno a lo que se denominó "sistema de información y conocimiento agrario" y tenían como base la concepción de los "sistemas blandos" (Checkland, 1993).

Tabla 1. Características seleccionadas de los productores ganaderos familiares y de sus establecimientos en Uruguay, en la región Sierra del Este y en el departamento de Maldonado.

\begin{tabular}{|c|c|c|c|}
\hline & País & Sierra del Este & Maldonado \\
\hline $\mathrm{N}^{0}$ de productores ganaderos familiares* & 22761 & 5375 & 1207 \\
\hline Tamaño promedio del establecimiento (ha) & 107 & 136 & 136 \\
\hline Tamaño promedio del rodeo de cría vacuna (cabezas)** & 34 & 49 & 44 \\
\hline Relación oveja de cría/vaca de cría & 2,21 & 2,21 & 2,53 \\
\hline \% de Campo natural & $72,2 \%$ & $79,8 \%$ & $75,8 \%$ \\
\hline Edad promedio (años) & 53 & 53 & 55 \\
\hline Nivel de educación*** & $44,1 \%$ & $49,7 \%$ & $50,1 \%$ \\
\hline$\%$ que reside en el predio & $60,7 \%$ & $47,6 \%$ & $48,1 \%$ \\
\hline$\%$ que recibe asistencia técnica & $38,4 \%$ & $32,5 \%$ & $27,6 \%$ \\
\hline
\end{tabular}

*Se toman como productores ganaderos familiares, aquellas personas físicas (condición jurídica) que explotan vacunos con explotaciones de hasta 500 ha y no desarrollan lechería comercial.

**Dado que se han promediado todos los establecimientos, para los sistemas criadores este valor esta subvalorado. ***El porcentaje que se presenta se calculó considerando todos los titulares (personas físicas) que han hecho cursos de educación formal de nivel secundario o superior. Elaborado a partir de datos del DIEA(2013) 
La conceptualización de los Sistemas de Innovación Agrarios mantiene la centralidad del vínculo investigación-extensión (clásico en los diferentes paradigmas y enfoques), a la vez que resalta la importancia de los relacionamientos sinérgicos entre todos los actores relevantes del sistema (más allá de la investigación, extensión y los productores) incluyendo al sector privado y al mercado. Al aumentar el número de actores del sistema, las chances de que existan visiones contrapuestas o no alineadas aumentan. Por eso los SIA necesitan construir una visión de futuro consensuada, una especie de pacto que implica "tejer la trama" en el marco de una red o plataforma. La literatura, Klerkx et al. (2009), cita la existencia de brechas entre los actores (por ej. diferentes incentivos generando brechas económicas) como un obstáculo al desarrollo de estos grupos heterogéneos, llamados también red o plataforma (Tabla 2).

Tabla 2. Principales fallas reportadas en los Sistemas de Innovación Agrarios.

Física: caminería, telecomunicaciones, etc

Fallas de infraestructura

Conocimiento: centros de investigación carentes de laboratorio y edificios. Financieras: es una limitante central para cualquier inversión que se acople a un proceso de innovación.

Falta de protección de propiedad intelectual

Fallas institucionales "duras" Falta de legislación en favor de tecnologías existentes Incentivos inadecuados.

Fallas institucionales blandas Falta de una cultura de innovación y colaboración, y emprendedora

Redes débiles: Falta una visión compartida

Redes duras: Alianzas establecidas generan sistemas cerrados a la interacción con otros actores

Fallas en la interacción

Limitantes en las capacidades existentes (ej., educación) para gestionar

Falla de capacidades innovaciones

Fuente: adaptado de Klerkx et al. (2012)

Este enfoque destaca fuertemente: i) la interacción entre la investigación y la actividad económica, ii) las actitudes y prácticas que promueven la interacción y el aprendizaje que las rodea, iii) la creación de un ambiente facilitador que coopere con la interacción y la innovación (Klerkx et al., 2012).

Los sistemas de innovación deben cumplir diferentes funciones, las principales según (Klerkx et al., 2009) son:

- Creación, intercambio y uso de conocimiento

- Fomento de la actividad y el espíritu emprendedor

- Desarrollo de una visión de futuro

- Movilización de recursos (capital financiero, humano)

- Formación de mercados (crear o adaptar)

- Promoción del cambio (superar resistencia al cambio)

El Programa Cooperativo para el Desarrollo Agroalimentario y Agroindustrial del Cono Sur (Catullo et al., 2014), señala cuales son los actores del Sistema de
Innovación: subsistema de ciencia, tecnología y extensión; productores; redes y organizaciones de productores y empresas; organizaciones de la comunidad; y diferentes niveles de Gobierno. Como dice el documento de Catullo et al. (2014) la acción de la Asistencia Técnica y Extensión Rural (ATER) “está orientada a la dinamización de espacios donde el intercambio de información y conocimientos impulsa procesos de innovación, respondiendo a las necesidades de un territorio como construcción social, identificando las oportunidades de transformación e incorporando los saberes e intereses locales. El objetivo es incrementar las capacidades de gestión de los recursos disponibles para alcanzar el desarrollo sustentable, donde el individuo pasa a ser el sujeto y no el objeto de las acciones e intervenciones". A tales efectos continúa siendo prioritario "profundizar esquemas y dispositivos de articulación efectiva entre los diferentes sistemas de investigación y asistencia técnica y extensión de acuerdo con las características, historia e instituciona- 
lidad de cada país". No obstante, esta relación entre la investigación y ATER no puede reproducir la clásica mera intermediación de información entre investigadores y los productores. Los equipos de ATER deben ser "facilitadores de procesos de innovación codiseñada horizontalmente".

Es importante entender el SIA en un sentido más amplio que incluye otras dimensiones en lo que es el desarrollo a nivel de una región. Para Boisier (1999), existen cuatro perspectivas a considerar: i) la política, en donde se expresa la capacidad regional para tomar las decisiones relevantes con relación a diferentes opciones de desarrollo y a la capacidad de diseñar y ejecutar políticas de desarrollo y la capacidad de negociar, ii) la económica, que se refiere a la apropiación y reinversión regional de parte del excedente para generar una base permanente de sustentación en el largo plazo, iii) la científica y tecnológica, como la capacidad regional para generar sus propios impulsos tecnológicos de cambio, y iv) la cultural, que se vincula con la identidad socio territorial. La dimensión del SIA se relaciona al plano científico tecnológico, pero debe verse en interacción con las restantes dimensiones.

El trabajo que se presenta en este artículo, forma parte del primer componente del proyecto "Co-innovación para el desarrollo de la ganadería familiar en la región de Sierra del Este", FPTA 343. Este componente tiene por objetivo caracterizar el sistema de innovación de los productores ganaderos familiares ubicados en dicha región, principalmente en el Departamento de Maldonado (región de intervención del mencionado proyecto). Por razones de espacio, hemos dividido el trabajo en dos partes, la primera, que estamos presentando, incluye la caracterización de los actores del sistema. En una segunda contribución presentaremos un análisis del funcionamiento del sistema desde la perspectiva de los productores, técnicos extensionistas e investigadores.

\section{MATERIALES Y MÉTODOS}

El estudio del SIA en la región analizada se efectúa en el marco de la implementación del proyecto FPTA
343. El fin del proyecto es "Contribuir a la coinnovación para el desarrollo sustentable de la ganadería familiar en la región de Sierra del Este y áreas de influencia”. El propósito se define como lograr el "Fortalecimiento de organizaciones de la ganadería familiar en Sierra del Este". La estrategia de intervención del proyecto define tres espacios de trabajo:

1) El predio ganadero, mediante procesos de planificación conducidos a través de instancias de capacitación-acción con los productores.

2) El grupo de productores, como espacio de asistencia técnica, capacitación e interacción entre los productores y técnicos.

3) La articulación con las instituciones de referencia, como espacio para otorgar factibilidad a tecnologías de altos requerimientos de escala (uso de maquinaria, operativo de silo de grano húmedo de sorgo, etc.) y competitividad en la comercialización de insumos y productos.

El proyecto se estructura en torno a cinco componentes: 1) Caracterización del sistema de innovación regional, 2) Agrupamiento y capacitación de productores, 3) Identificación de tecnologías de articulación asociativas, 4) Integración a cadenas de valor, y 5) Seguimiento y Evaluación. Se pueden identificar cuatro tipos de problemas cuando se trabaja con la producción familiar: i) los problemas que se solucionan desde la $\mathrm{I}+\mathrm{D}$, ii) los problemas que se solucionan con ATER (cuando existe una brecha entre el potencial productivo a nivel de investigación y de las practicas que realizan los productores, en este caso de la ganadería familiar), iii) los problemas estructurales (por ej., tamaño de las explotaciones, que pueden enfrentarse con tecnologías asociativas), y iv) los problemas vinculados al mercado y/o comercialización (Albin 2017). El proyecto FPTA 343 implementa acciones que corresponden a los puntos 2, 3 y 4 antes mencionados (a través de los componentes 2, 3 y 4 , respectivamente). 


\section{Aspectos metodológicos}

A los efectos de estudiar el SIA en la región se utilizaron las siguientes técnicas: entrevistas semiestructuradas a informantes calificados y talleres de discusión con productores. El cuestionario de las entrevistas fue diseñado por el equipo técnico del proyecto FPTA 343, considerando los distintos perfiles de los actores a consultar. Entre 40 y 80 minutos fue la duración de las entrevistas dependiendo del informante consultado, fueron grabadas bajo consentimiento y luego desgrabadas. Las entrevistas semiestructuradas se realizaron siguiendo la técnica de "bola de nieve", mediante un proceso de acumulación hasta lograr un punto de saturación en el cual se considera que se han captado todas las dimensiones de interés, y que nuevas entrevistas no aportan información relevante al estudio.

También se utilizaron talleres de discusión para relevar los vínculos de las organizaciones de productores ganaderos en el marco del SIA. Las técnicas utilizadas en estos talleres fueron: i) construcción de mapas parlantes en subgrupos, y ii) diagrama de Venn para elaborar mapas de actores e identificar los principales relacionamientos y articulaciones que tienen las organizaciones con los demás actores sociales de la región (Geilfus 2002). También se detectaron necesidades de capacitación y se relevaron los principales problemas que tienen las organizaciones. El trabajo de campo se realizó durante el 2017.

\section{RESULTADOS Y DISCUSIÓN}

En este punto se aportan elementos para la discusión en torno a la identificación de los actores principales del SIA. Es necesario advertir que la región Este (Departamentos de Lavalleja, Maldonado, Rocha, Treinta y Tres y parte de Cerro Largo) abarca un área mayor al Departamento de Maldonado (base del proyecto FPTA); esto implica que algunas consideraciones de este capítulo pueden resultar relativamente más válidas para la región en su conjunto que estrictamente para el área de intervención del proyecto (no obstante no alteran las tendencias básicas).

\section{Actores}

INIA Treinta y Tres

El INIA Treinta y Tres, una de las cinco sedes regionales del Instituto Nacional de Investigación Agropecuaria (INIA). Centra su trabajo en las siguientes áreas: sistema de producción arroz con pasturas (donde se aprovecha el pastoreo de ganado vacuno y ovino), producción y evaluación de pasturas sembradas y campo natural, es sede de los programas nacionales de investigación de Arroz y Producción y sustentabilidad ambiental, se realizan acciones vinculadas a los programas de producción de Carne y Lana, Pasturas y Forrajes, Hortícola, Frutícola y Agricultura Familiar, y tiene localizada una unidad de semillas y biotecnología y evaluación de cultivares. El INIA Treinta y Tres además de su sede central ubicada en el km 282 de ruta 8 , donde está radicada su administración y dirección, posee tres campos o unidades experimentales: semillas (semilleros), Palo a Pique (énfasis en ganadería vacuna y ovina, pasturas, malezas), y Paso de la Laguna (cultivo de arroz y pasturas) (INIA, 2018).

El INIA Treinta y Tres es el actor líder en la generación de conocimiento de la región, su área de acción involucra una extensión de más de 4 millones de hectáreas en la región este, abarcando los departamentos de Treinta y Tres, Lavalleja, Rocha, Maldonado y parte de Cerro Largo que comparte con INIA Tacuarembó. No obstante, la misma institución manifiesta que es un área muy extensa a cubrir reconociendo que hay territorios donde le cuesta llegar y tiene dificultades para mantener presencia.

Otra preocupación hace referencia a que las líneas de investigación reflejen realmente las necesidades de los productores. En este sentido INIA tiene dos ámbitos formales donde los productores participan, uno es el CAR (Consejo Asesor Regional) que es un órgano de asesoramiento al director de la regional (actualmente participan delegados en ganadería de todos los deptos. donde tiene influencia esta sede). El otro ámbito son los GT (Grupos de Trabajo) que constituyen un espacio donde se discuten aspectos de las líneas de investigación, está integrado por investigadores, extensionis- 
tas y productores delegados. En este último ámbito, la situación actual para el rubro ganadero refleja dificultades de trabajo frecuente, con inestabilidad en las reuniones del GT, es incierta la participación de actores externos al INIA y los delegados carecen de representatividad, participando muchas veces a título personal.

Existe un espacio a explorar de interacción con iniciativas de la región en el marco de proyectos. En los últimos tiempos la región Este (al igual que en la región del Basalto, zona norte y centro del Uruguay) ha convocado muchas iniciativas estructuradas en proyectos que por su misma lógica desarrollan estrategias que pueden no favorecer los relacionamientos a nivel de los territorios. En la región de Maldonado se realizaron acciones de un proyecto basado en el uso de los predios foco, donde intervinieron INIA, MGAP, IPA, Agricultural Research (de Nueva Zelanda) y organizaciones de productores ganaderos.

Actualmente se lleva adelante un proyecto "más tecnologías" integrando a INIA, CALIMA y la Escuela Agraria de San Carlos. El mismo tiene por objetivo evaluar el comportamiento de variedades de Festuca en seis parcelas distintas en el departamento de Maldonado. La metodología aplicada consiste en el manejo de parcelas demostrativas en predios comerciales y en el predio de la Escuela agraria. Comenzó en abril de 2017 y en diciembre del mismo año se realizó una actividad abierta a productores y técnicos de la región mostrando los primeros resultados

Instituto Plan Agropecuario (IPA)

La regional Este del IPA está conformada por 4 técnicos, uno por cada departamento (Maldonado, Rocha, Lavalleja y Treinta y Tres); tres son ingenieros agrónomos y una veterinaria. El equipo cumple con una agenda anual coordinada de temas que se ajustan al ciclo de producción ganadero. En este sentido hay actividades que ya son clásicas, como la gira de rodeo de cría y la gira de pasturas. Se trabaja coordinadamente con otras instituciones como ser INIA (se valora como intensa), SUL, MGAP y empresas privadas que participan.

También se mantiene un relacionamiento activo con un número muy importante de organizaciones de productores. El relacionamiento es con sociedades de fomento, sociedades agropecuarias, cooperativas y grupos de productores. A partir de este relacionamiento activo se identifican las demandas de acciones de extensión. Las acciones de extensión van desde charlas temáticas (manejo del rodeo, manejo del campo natural, gestión de empresa, etc.), pasando por cursos

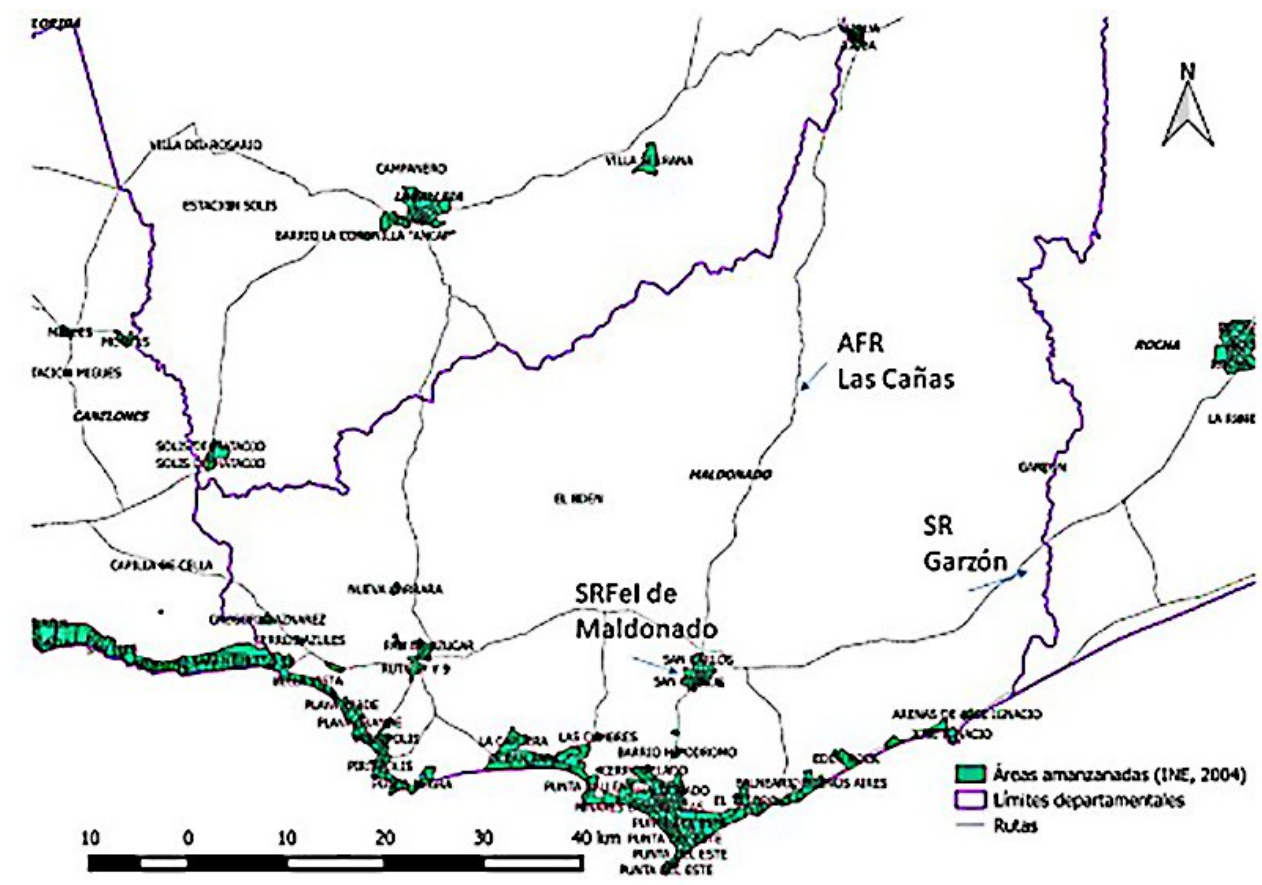

Figura 1. Mapa de la región de Maldonado donde se está realizando el proyecto Fpta 343. 
presenciales y a distancia, como también giras y visitas a otras zonas del territorio de la región como de extra región.

\section{Las Organizaciones de productores}

Las organizaciones de productores ganaderos que ocupan particular relevancia para este proyecto (FPTA 343) son la Sociedad de Fomento Rural (SFR) Garzón, la Asociación de Fomento Rural (AFR) Las Cañas y la SFR e Industrial de Maldonado (Figura 1).

Para analizar el funcionamiento de estas organizaciones se efectuaron tres talleres de diagnóstico rural rápido con cada una de las organizaciones de productores. Se utilizó el diagrama De Venn como herramienta a los efectos de identificar los principales relacionamientos y articulaciones con los distintos actores que participan en el SIA del sector ganadero de la región este del Uruguay.

Para la realización de este Diagrama los socios de cada organización listaron aquellas organizaciones u actores que consideran relevantes para la Sociedad de Fomento. Posteriormente ubicaron en un esquema a estas organizaciones y actores con respecto a la SFR teniendo en cuenta 2 criterios: i) de cercanía, significando el grado de relacionamiento con la SFR, y ii) de tamaño del círculo con el que se identificaba cada uno, significando el grado de importancia que la SFR le adjudica.

A continuación se presentan las figuras que muestran los resultados obtenidos.

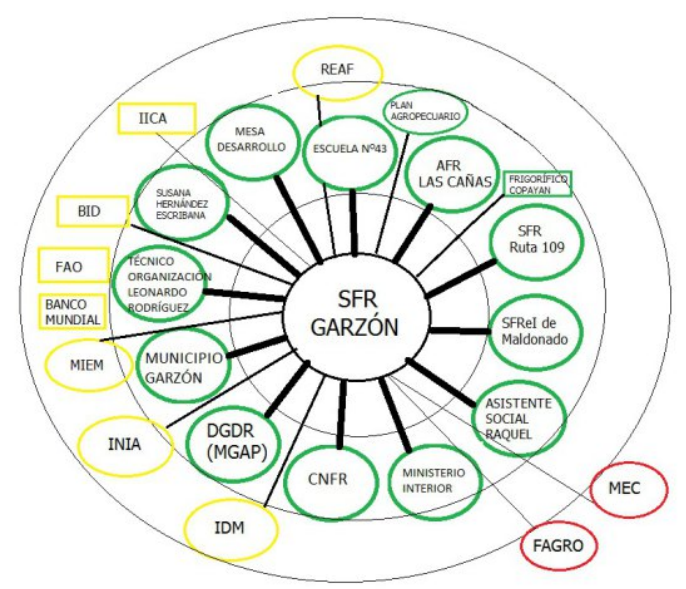

Figura 2. Mapa de actores y sus relación con la Sociedad de Fomento Rural Garzón. (Circulo verde mayor nivel de relación, en amarillo intermedio, y en rojo relación reciente o de poca relevancia).
En la figura 2, se destaca que el diagrama de esta organización tanto por cercanía como por importancia la Dirección General de Desarrollo Rural del MGAP (DGDR), así como los técnicos de la organización (Leonardo Rodriguez, etc.). Esto se explica claramente por el reconocimiento al apoyo recibido por la DGDR y la valoración del papel de los técnicos en la SFR

También aparecen con relevancia otras organizaciones de la zona (SFR Las Cañas, SRF e Industrial de Maldonado, SFR Ruta 109) lo que indica un sentido de pertenencia e identificación a un grupo mayor. Lo cual también sucede con la Comisión Nacional de Fomento Rural(CNFR) que las integra. Esto es un signo positivo en cuanto al reconocimiento de instancias grupales regionales, lo que genera condiciones para acciones conjuntas. Otro actor que aparece con destaque es la Mesa de Desarrollo Rural del departamento.

En este caso hay una mención a una industria local (Frigorífico Copayan de Rocha) dado que la Sociedad de Fomento tiene con el mismo un acuerdo comercial.

El Instituto Plan Agropecuario aparece con identificación importante en cuanto a relacionamiento pero con valoración menor en cuanto a importancia para la organización. El INIA y FAGRO importantes en un sistema de innovación, aparecen en el Diagrama, pero con poca relevancia en cuanto a su relacionamiento con la institución y también con un grado relativo de importancia.

Un actor normalmente importante en lo local como la Intendencia Municipal (IDM), aparece, pero con escasa valoración en cuanto al grado de relacionamiento. En este aspecto se contrapone con la importancia asignada al Municipio local de Garzón.

En la figura 3, se destaca que el diagrama de esta organización por su relacionamiento e importancia atribuida la Mesa de Desarrollo Rural, el MGAP, la Sociedad de Fomento Rural de Garzón y la Sociedad de Fomento Rural e Industrial de Maldonado. En el caso de estas últimas la valoración puede responder a su integración en elFPTA343.

El Plan Agropecuario aparece con relevancia pero en un $2^{\circ}$ nivel. En ese $2^{\circ}$ nivel de relacionamiento aparece 


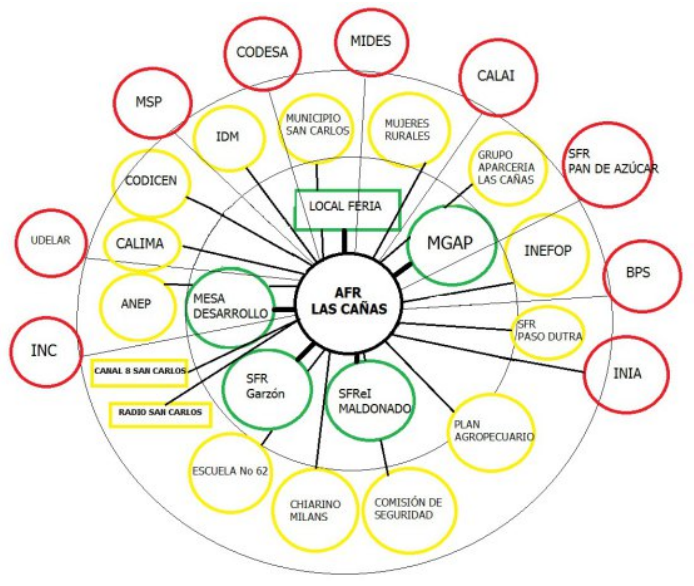

Figura 3. Mapa de actores y sus relación con la Asociación de Fomento Rural Las Cañas. (Circulo verde mayor nivel de relación, en amarillo intermedio, y en rojo relación reciente o de poca relevancia)

otra organización del sector como CALIMA, dado que varios de los socios de la AFR también lo son de esta organización, pero este relacionamiento se basa fundamentalmente en temas comerciales.

Aparecen menciones también en este nivel a otras organizaciones rurales de la Zona como La SFR de Paso Dutra y la SFR Chiarino Milans. Coincidentemente con lo visto en la SFR Garzón, en Las Cañas la Intendencia Departamental de Maldonado (IDM) aparece con menor valoración tanto en grado de relacionamiento como de importancia, que el Municipio local de San Carlos. Esto puede hablar de una escasa participación de la Intendencia así como de un buen relacionamiento con el municipio.

Se destaca en esta organización la valoración dada a la capacitación, con referencias a la Escuela $N^{0} 61$, ANEP, CODICEN, INEFOP organizaciones con las que entienden debería ampliarse el vínculo (actores del sistema de innovación).

En el caso de INIA si bien lo ubican lejos en cuanto a relacionamiento, le otorgan importancia y hubo manifestaciones expresas en cuanto a que entienden que dicha institución debería tener más proximidad (objetivo del proyecto). La UDELAR aparece con escaso relacionamiento, dado que no había participación en la zona hasta el comienzo del FPTA 343.

En la figura 4, se observa que el diagrama de esta organización, identifican alto relacionamiento con actores locales como la Intendencia municipal de

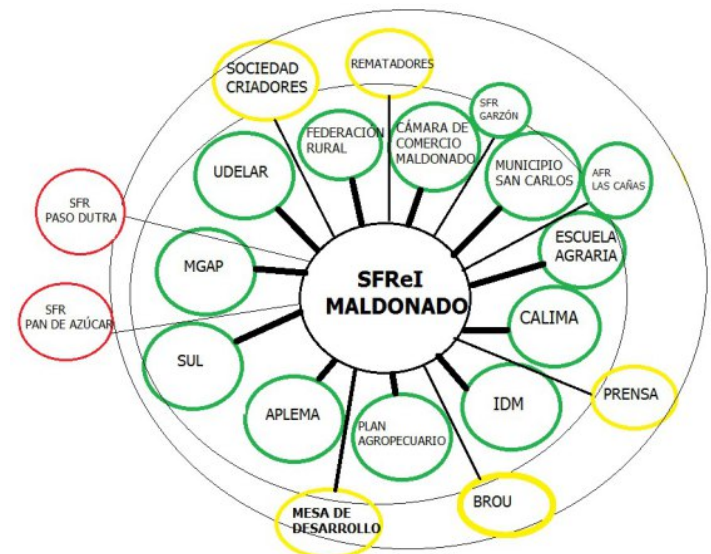

Figura 4. Mapa de actores y sus relación con la Sociedad de Fomento Rural e Industrial de Maldonado. (Circulo verde mayor nivel de relación, en amarillo intermedio, y en rojo relación reciente o de poca relevancia)

Maldonado(IDM), el municipio local, Escuela Agraria de San Carlos, Consejo de Educación Técnica y Profesional, CALIMA, APLEMA y también otros de carácter nacional como MGAP, INIA, SUL, IPA, UDELAR . También atribuyen importancia a estos relacionamientos.

La referencia a CALIMA, al igual que en la AFR Las Cañas, responde a la participación de varios de sus socios en ambas instituciones. A diferencia de lo visto en los diagramas de las otras organizaciones, en este diagrama aparece con alto grado de relacionamiento e importancia la Intendencia Departamental de Maldonado, en un mismo nivel que el municipio local. Es de resaltar la importancia atribuida a organismos de generación y transferencia como INIA, IPA, SUL y UDELAR actores relevantes en el sistema. En esta organización aparecen como relevantes instituciones como la Federación Rural, por su integración a la misma y Sociedades de Criadores de distintas razas dada la importancia del papel de esta organización en la organización de ferias ganaderas.

Con alto relacionamiento pero un nivel menor de importancia aparecen también las otras sociedades de fomento integrantes del proyecto FPTA 343. También en contraposición con lo visto en los otros diagramas, la Mesa de Desarrollo aparece con un menor grado de relacionamiento con la SFR, aunque con valoración en cuanto a su importancia.

Del análisis de los mapas de actores surgen las 
siguientes reflexiones:

Las tres organizaciones cuentan con relacionamientos potenciales para desencadenar procesos de desarrollo, en sus áreas de influencia.

- Las tres organizaciones reconocen vínculos entre ellas, que permite pensar en un abordaje regional y no solo local. Con una importante reconocimiento entre las que comparten el FPTA 343, pero también con otras organizaciones de la zona.

- En dos de los tres casos, los agentes de la investigación (INIA y Fagro-UdelaR) aparecen con poco vínculo, esto es relevante ya que son actores importantes en el sistema de innovación.

- El IPA es reconocido como agente de transferencia y aparece con mayor relevancia en los diagramas.

- La mesa de desarrollo rural es un espacio reconocido y destacado, al igual que el MGAP, en particular a través de la llegada de políticas de apoyo canalizadas por la DGDR. Estas políticas explican el \% de servicios de ATER que reciben los productores (en la línea de base de INIA para la evaluación del proyecto el $42 \%$ recibe ATER; $\sin$ estas políticas el valor seria a nuestro modo de ver significativamente inferior).

- Se destaca la poca vinculación y reconocimiento de la Intendencia departamental (IDM) en SFR Garzón y AFR Las Cañas. Así como el mayor relacionamiento con los municipios locales.

- Sólo SFR Garzón se vincula a una industria frigorífica(COPAYAN).

Las valoraciones y reflexiones que surgen de este trabajo se entiende coinciden en líneas generales con la línea de base desarrollada por INIA para evaluar el proyecto.

\section{Problemas}

Se identifican los siguientes problemas que afectan el relacionamiento entre los actores:

- Debilidades en las organizaciones de productores: se observaron problemas de convocatoria (algunos relacionados con falta de sede), falta de participación de jóvenes, concentración de responsabilidades y tareas en pocas personas, escasa articulación con las agroindustrias de la región y con los proveedores locales y dificultades para el planteo de demandas a los centros de investigación, dificultades para mantener avances logrados en proyectos previos (DGDR/MGAP) cuando los mismos finalizan

- Debilidades en INIA para mantener ámbitos de captación de demandas de investigación

- Debilidades de los actores vinculados a la asistencia técnica y extensión rural (básicamente IPA) derivadas de la relación recursos humanos existentes y población objetivo a atender

\section{CONCLUSIONES}

La caracterización del SIA de la región Este con énfasis en el área de influencia del proyecto Fpta 343 para el desarrollo de la ganadería familiar presenta las siguientes características.

- Los principales actores del SIA son: a) actores institucionales: INIA 33 e IPA b) organizaciones de productores: SFR Garzón, AFR Las Cañas y SFR e Industrial de Maldonado.

- A estos actores se suman el Frigorífico Copayan y CALIMA, quienes pueden desempeñar roles positivos en la gestión del conocimiento desde la demanda de productos y desde la oferta de insumos para la producción ganadera.

- Espacios de coordinación como la Mesa de Desarrollo Rural, de reciente funcionamiento, si bien involucran a las organizaciones de productores y otros actores institucionales, como DGDRMGAP, aunque no parecen tener importantes efectos en estimular las interacciones del SIA ganadero a nivel departamental.

- Desde el área de la producción no aparece claro la presencia de interlocutores organizados, que interactúen con el resto de los componentes del sistema (investigación y extensión en particular, pero tampoco con el mercado). Es necesario mejorar los niveles de capital social de las organizaciones de productores ganaderos y su capacidad de participación en estructuras de decisión. La instancia de coordinación Mesa de 
Desarrollo Rural puede jugar un papel en este sentido.

- Desde la investigación los dispositivos para captar demandas de los productores en la actualidad no funcionan de la mejor forma. Los grupos de trabajo han caído en una suerte de desuso y no son convocados en algunos casos desde hace ya 2 años; existen debilidades propias de este espacio de interacción entre productores, investigadores y técnicos de campo (no tiene una dinámica de trabajo frecuente y estable, es incierta la participación de actores externos al INIA, y carecen de representatividad los delegados). El CAR tiene una dinámica de funcionamiento con algunas reuniones anuales, pero con escasa participación de representantes en el rubro ganadero, lo que desalienta el planteo de demandas para este sector (los investigadores entienden que hay un problema de ausencia de interlocutores organizados en la ganadería del este); en cambio los representantes del sector arrocero, ejercen presión, demandan y continuamente motivan el funcionamiento del espacio de discusión.

\section{V.AGRADECIMIENTOS}

Los autores desean agradecer muy especialmente a los productores y técnicos pertenecientes a las organizaciones que participan en el proyecto por sus aportes realizados para la elaboración de este estudio. Así mismo a todas las personas que oficiaron como informantes calificados y dedicaron su tiempo para poder realizar las entrevistas. Finalmente, al INIA por financiar estas actividades del proyecto.

\section{REFERENCIAS BIBLIOGRÁFICAS}

Albin, A. 2017. “Investigación, Desarrollo e Innovación Para La Agricultura Familiar.” Revista INIA 50 (9): 41-46.

Boisier, S. 1999. El Desarrollo Territorial a Partir de La Construcción Del Capital Sinergético. Una Contribución Al Tema Del Capital Intangible Del Desarrollo. Instituciones y
Actores Del Desarrollo Territorial En El Marco de La Globalización. Santiago de Chile (Chile): Ediciones Iniversidad del BioBio.

Catullo, J. C., H. G. Varela, C. Alemany, G. Torres, F. Chávez, L. Brunel, O. Balsadi, et al., 2014. Rol De La Extensión Rural En La Gestión De Innovaciones. Montevideo (Urugay): PROCISUR.

Checkland, P. 1993. Pensamiento de Sistemas, Practica de Sistemas. Mexico D.F. (México): Editorial Limusa.

DIEA. 2013. Censo General Agropecuario 2011: Resultados Definitivos. Montevideo (Urugay): Ministerio Ganadería, Agricultura y Pesca.

Geilfus, F. 2002. 80 Herramientas Para El Desarrollo Participativo. San José(Costa Rica): IICA.

Gómez, R., and H. Saravia. 2016. “Tecnología En Sistemas Ganaderos Criadores de Sierras Del Este: Oferta Disponible y Toma de Decisiones Tecnológicas En El Predio.” Agrociencia Urugua y 20 ( 1 ): $113-22$. http://www.scielo.edu.uy/scielo.php?script=s c i_a r t t e x t \& p i d = S 2301 15482016000100014\&lang=pt.

INIA. 2015. Bases 2015 Para La Presentación de Propuestas de Promoción Tecnológica Fortaleciendo La Co-Innovación. Montevideo (Urugay): Instituto Nacinal de Investigación Agropecuaria.

I N I A. 2018 . INIA treinta y tres. ht tp://www.inia.uy/estacionesexperimentales/direcciones-regionales/iniatreinta-y-tres

Klerkx, L., A. Hall, and Cees Leeuwis. 2009. "Strengthening Agricultural Innovation Capacity: Are Innovation Brokers the Answer?" International Journal of Agricultural Resources, Governance and E c o l o g y 8 ( 5 - 6) : 409 . doi:10.1504/IJARGE.2009.032643. 
Klerkx, L., B. Van Mierlo, and Cees Leeuwis. 2012. "Evolution of Systems Approaches to Agricultural Innovation: Concepts, Analysis and Interventions." En Farming Systems Research into the 21st Century: The New Dynamic. Darnhofer, I., D. Gibbon, y B. Dedieu (eds). Londre (Reino Unido): Springer. doi:10.1007/978-94-007-4503-2. 